\title{
A partial converse to the Andreotti-Grauert theorem
}

\author{
Xiaokui Yang
}

\begin{abstract}
Let $X$ be a smooth projective manifold with $\operatorname{dim}_{\mathbb{C}} X=n$. We show that if a line bundle $L$ is $(n-1)$-ample, then it is $(n-1)$-positive. This is a partial converse to the Andreotti-Grauert theorem. As an application, we show that a projective manifold $X$ is uniruled if and only if there exists a Hermitian metric $\omega$ on $X$ such that its Ricci curvature $\operatorname{Ric}(\omega)$ has at least one positive eigenvalue everywhere.
\end{abstract}

\section{Contents}

1. Introduction 1

2. Partially positive line bundles on compact complex manifolds 4

3. The pseudo-effective line bundles on compact complex manifolds 6

4. The proofs of Theorem 1.4 and Theorem $1.5 \quad 8$

$\begin{array}{ll}\text { References } & 11\end{array}$

\section{Introduction}

One of the most fundamental topics in algebraic geometry is the characterizing ampleness of line bundles by using numerical and cohomological vanishing theorems. The theorem of Cartan-Serre-Grothendieck is a milestone in this direction: the following statements are equivalent

(1) $L$ is an ample line bundle over a projective manifold $X$;

(2) For every coherent sheaf $\mathcal{F}$ on $X$, there exists a positive integer $m_{0}=m_{0}(X, \mathcal{F}, L)$ such that

$$
H^{i}\left(X, \mathcal{F} \otimes L^{\otimes m}\right)=0
$$

for all $i>0$ and all $m \geq m_{0}$.

On complex projective manifolds, ampleness is also equivalent to the existence of a smooth metric with positive curvature, thanks to the celebrated Kodaira embedding theorem.

In [AG62], Andreotti and Grauert considered the case of line bundles with curvature of mixed signature. Now it is formulated into 
Definition 1.1. Let $L$ be a holomorphic line bundle over a compact complex manifold $X$ with $\operatorname{dim}_{\mathbb{C}} X=n$.

(1) $L$ is called q-positive, if there exists a smooth Hermitian metric $h$ on $L$ such that the Chern curvature $R^{(L, h)}=-\sqrt{-1} \partial \bar{\partial} \log h$ has at least $(n-q)$ positive eigenvalues at every point on $X$.

(2) $L$ is called $q$-ample, if for any coherent sheaf $\mathcal{F}$ on $X$ there exists a positive integer $m_{0}=m_{0}(X, L, \mathcal{F})>0$ such that

$$
H^{i}\left(X, \mathcal{F} \otimes L^{m}\right)=0 \quad \text { for } i>q, m \geq m_{0} .
$$

It is obvious that $L$ is 0 -positive if and only if $L$ is positive, and $L$ is 0 -ample if and only if $L$ is ample. Hence the 0-positivity and 0-ampleness are equivalent. In [AG62, Theorem 14], Andreotti and Grauert proved the following fundamental theorem (see also [DPS96, Proposition 2.1]).

Theorem 1.2 (Andreotti-Grauert). A q-positive line bundle is q-ample.

Historically, the Andreotti-Grauert Theorem is the first result on the relationship between (partially) positive line bundles and the cohomological vanishing theorems. In the pioneer work [DPS96], Demailly, Peternell and Schneider systematically investigated partial vanishing theorems and proposed the following problem on the converse to the Andreotti-Grauert theorem:

Problem 1.3. On a projective manifold, if a line bundle is $q$-ample, is it $q$-positive?

This is a long-standing open problem. The key difficulty arises from constructing a precise metric according to the formal partial vanishing theorem (1.1). Recently, there are some progress on this problem, mainly contributed by Demailly, Totaro, Ottem, Küronya, Matsumura, Brown and etc (see [Dem11, Tot13, Mat13, Ott12, Bro12, Kür10, GK15] and also the references therein). Totaro proved in [Tot13] that the notion of $q$-ampleness is equivalent to others previously studied in [DPS96]. As a result, the $q$-ampleness of a line bundle depends only on its numerical class, and the cone of such bundles is open. In particular, Totaro established that the $(n-1)$ ample cone of an $n$-dimensional projective manifold $X$ is equal to the negative of the complement of the pseudo-effective cone of $X$ (see also Corollary 1.6). In dimension two, Demailly proved in [Dem11] an asymptotic version of this converse to the Andreotti-Grauert Theorem using tools related to the holomorphic Morse inequality and asymptotic cohomology; subsequently, Matsumura obtained in [Mat13, Theorem 1.3] a positive answer to the question for projective surfaces. However, there exist higher-dimensional counterexamples to the converse Andreotti-Grauert problem in the range $\frac{\operatorname{dim} X}{2}-1<q<\operatorname{dim} X-2$, constructed by Ottem [Ott12, Theorem 10.3]. Our main result in this paper is a partial converse to the Andreotti-Grauert theorem on smooth projective manifolds. 
Theorem 1.4. Let $L$ be a line bundle over a smooth projective manifold $X$ with $\operatorname{dim}_{\mathbb{C}} X=n$. If $L$ is $(n-1)$-ample, then it is $(n-1)$-positive.

In particular, when $\operatorname{dim}_{\mathbb{C}} X=2$, the converse Andreotti-Grauert Problem 1.3 is true (see also [Mat13, Theorem 1.3]). Actually, Theorem 1.4 is a straightforward application of the following result on general compact complex manifolds.

Theorem 1.5. Let $X$ be a compact complex manifold with $\operatorname{dim}_{\mathbb{C}} X=n$. Then the following statements are equivalent:

(1) $L$ is $(n-1)$-positive;

(2) the dual line bundle $L^{-1}$ is not pseudo-effective.

Note that, Theorem 1.5 is also valid if we replace the line bundle $L$ by a Bott-Chern class $\alpha \in H_{\mathrm{BC}}^{1,1}(X)$ (see Theorem 4.2). The key ingredients in the proof of Theorem 1.5 rely on several results in our previous paper [Yang17] on geometric characterizations of pseudo-effective line bundles (resp. Bott-Chern classes). As an application of Theorem 1.4, Theorem 1.5 and Theorem 1.2, one has

Corollary 1.6. On a projective manifold $X$ of complex dimension $n$, the following are equivalent:

(1) $L$ is $(n-1)$-ample;

(2) $L$ is $(n-1)$-positive;

(3) $L^{-1}$ is not pseudo-effective.

Note that the equivalence of (1) and (3) is also obtained in [DPS96, Proposition 2.5], [Tot13, Theorem 9.1] and [Mat13, Lemma 2.4] by different methods in one direction.

According to Ottem's counter-examples in [Ott12, Theorem 10.3], for $\frac{\operatorname{dim} X}{2}-1<$ $q<\operatorname{dim} X-2$, the $q$-ampleness can not imply the $q$-positivity. On the contrary, by Theorem 1.4, we obtain

Proposition 1.7. Let $X$ be a smooth projective manifold with $\operatorname{dim}_{\mathbb{C}} X=n$. Suppose $L$ is q-ample, then the restriction of $L$ to every codimension- $(n-q-1)$ smooth submanifold $Y$ is q-positive.

In particular, we have

Corollary 1.8. Let $X$ be a smooth projective manifold with $\operatorname{dim}_{\mathbb{C}} X=n$. If $L$ is $(n-2)$-ample, then the restriction of $L$ to every codimension-1 smooth submanifold is $(n-2)$-positive.

On the other hand, by using the classical result of [BDPP13] and Yau's theorem [Yau78], one obtains

Corollary 1.9. On a projective manifold $X$ of complex dimension $n$, the following are equivalent:

(1) $X$ is uniruled; 
(2) $K_{X}^{-1}$ is $(n-1)$-positive;

(3) there exists a smooth Hermitian metric $\omega$ on $X$ such that its Ricci curvature $\operatorname{Ric}(\omega)$ has at least one positive eigenvalue everywhere;

(4) $K_{X}$ is not pseudo-effective;

(5) $K_{X}^{-1}$ is $(n-1)$-ample.

Remark 1.10. The equivalence of (1) and (3) is also conjectured by S.-T Yau.

Remark 1.11. From Theorem 1.4, Theorem 1.5, Corollary 1.6, Corollary 1.9, Theorem 4.1 and Theorem 4.2, one can derive and formulate various cone dualities on compact complex manifolds. The vector bundle analogous of the main results and applications are obtained in [Yang18].

Acknowledgement. I am very grateful to Professor Kefeng Liu for his support, encouragement and stimulating discussions over years. I would also like to thank Professors Junyan Cao, J.-P. Demailly, S. Matsumura, Yum-Tong Siu, Junchao Shentu, Xiaotao Sun, Valentino Tosatti, Jian Xiao and Xiangyu Zhou for some useful suggestions. The author would also like to thank the anonymous referees whose comments and suggestions helped improve and clarify the paper. This work was partially supported by China's Recruitment Program of Global Experts and NSFC 11688101.

\section{Partially positive line bundles on compact complex manifolds}

In this section, we deal with two different notions on $q$-positive line bundles over compact complex manifolds.

Definition 2.1. Let $\mathrm{X}$ be a compact complex manifold and $L$ be a holomorphic line bundle over $X$.

(1) $L$ is called q-positive, if there exists a smooth Hermitian metric $h$ on $L$ and a smooth Hermitian metric $\omega$ on $X$ such that the Chern curvature $R^{(L, h)}=$ $-\sqrt{-1} \partial \bar{\partial} \log h$ has at least $(n-q)$ positive eigenvalues at any point on $X$ (with respect to $\omega$ );

(2) $L$ is called uniformly q-positive, if there exists a Hermitian metric $h$ on $L$ nd a smooth Hermitian metric $\omega$ on $X$ such that the summation of any distinct $(q+1)$ eigenvalues (counting multiplicity) of the Chern curvature $R^{(L, h)}=$ $-\sqrt{-1} \partial \bar{\partial} \log h$ is positive at any point of $X$ (with respect to $\omega$ ).

The following result is obtained by changing the metric on the complex manifold and the background idea dates back to [AV65, Section 5].

Proposition 2.2. The following statements are equivalent: 
(1) $L$ is q-positive;

(2) $L$ is uniformly q-positive.

Proof. (2) $\Longrightarrow(1)$. Let $\omega$ be a Hermitian metric on $X$ and $h$ be a smooth Hermitian metric on $L$ such that $R^{(L, h)}=-\sqrt{-1} \partial \bar{\partial} \log h$ is uniformly $q$-positive. Let $\lambda_{1} \geq \cdots \geq$ $\lambda_{n}$ be the eigenvalues of $R^{(L, h)}$ with respect to $\omega$ over some coordinate chart. We have $\lambda_{n-q}>0$. Otherwise, the summation of $q+1$ eigenvalues $\lambda_{n-q}+\lambda_{n-q+1}+\cdots+\lambda_{n} \leq 0$.

$(1) \Longrightarrow(2)$. We assume that there exists a smooth Hermitian metric $h$ on $L$ such that the curvature $R^{(L, h)}=-\sqrt{-1} \partial \bar{\partial} \log h$ has at least $(n-q)$ positive eigenvalues at each point $p \in X$. Let $\omega_{0}$ be a fixed Hermitian metric on $X$. For simplicity, we denote by $R$ and $\Omega$ the local matrix representations of the matrices $R^{(L, h)}$ and $\omega_{0}$ respectively, in some local holomorphic frames of $X$. Let

$$
\lambda_{1}(z) \geq \cdots \geq \lambda_{n}(z)
$$

be the eigenvalues of $R^{(L, h)}$ with respect to $\omega_{0}$. It is obvious that $\lambda_{1}, \cdots, \lambda_{n}$ are eigenvalues of the matrix $R \Omega^{-1}$. Note that $R \Omega^{-1}$ represents a tensor in $\Gamma\left(X, \operatorname{End}\left(T^{1,0} X\right)\right)$, and so the eigenvalues $\lambda_{i}$ are independent of the choice of coordinates. Since $\lambda_{n-q}$ is a continuous function, we set

$$
\lambda_{0}=\frac{\log (n+1)}{\inf _{X} \lambda_{n-q}} .
$$

$\lambda_{0}$ is a positive number since $L$ is $q$-positive and $X$ is compact. We define a new Hermitian metric $\omega$ over $X$ with local matrix representation $\widetilde{\Omega}$ by the following formula

$$
\widetilde{\Omega}^{-1}=\Omega^{-1} \cdot\left(I d+\sum_{k=1}^{\infty} \frac{\lambda_{0}^{k}\left(R \Omega^{-1}\right)^{k}}{(k+1) !}\right) .
$$

Note that the matrix $\widetilde{\Omega}^{-1}$ is positive definite. Indeed, the eigenvalues of the matrix $I d+\sum_{k=1}^{\infty} \frac{\lambda_{0}^{k}\left(R \Omega^{-1}\right)^{k}}{(k+1) !}$ are given by

$$
1+\sum_{k=1}^{\infty} \frac{\lambda_{0}^{k} \lambda_{i}^{k}}{(k+1) !}=\frac{e^{\lambda_{0} \lambda_{i}}-1}{\lambda_{0} \lambda_{i}}>0, \quad \text { if } \quad \lambda_{i} \neq 0 .
$$

It is not hard to see that the Hermitian metric $\omega$ is globally well-defined on $X$. Let $\kappa_{1} \geq \cdots \geq \kappa_{n}$ be the eigenvalues of $R^{(L, h)}$ with respect to the new metric $\omega$, i.e. they are the eigenvalues of $R \widetilde{\Omega}^{-1}$. Note also that

$$
R \widetilde{\Omega}^{-1}=\lambda_{0}^{-1}\left(\sum_{k=0}^{\infty} \frac{\lambda_{0}^{k}\left(R \Omega^{-1}\right)^{k}}{k !}-I d\right) .
$$

A straightforward computation shows

$$
\kappa_{n-q}=\frac{e^{\lambda_{0} \lambda_{n-q}}-1}{\lambda_{0}} \quad \text { and } \quad \kappa_{n}=\frac{e^{\lambda_{0} \lambda_{n}}-1}{\lambda_{0}} .
$$


For any summation of $(q+1)$ (distinct) eigenvalues of $R^{(L, h)}$ with respect to the new metric $\omega$, we have the inequality

$$
\begin{aligned}
\sum_{\ell=1}^{q+1} \kappa_{i_{\ell}} & \geq \kappa_{n-q}+\cdots+\kappa_{n} \\
& \geq \kappa_{n-q}+q \kappa_{n} \\
& \geq \lambda_{0}^{-1}\left(e^{\lambda_{0} \lambda_{n-q}}+q e^{\lambda_{0} \lambda_{n}}-(q+1)\right) \\
& >\lambda_{0}^{-1}\left(e^{\lambda_{0} \lambda_{n-q}}-(q+1)\right)>0
\end{aligned}
$$

since $e^{\lambda_{0} \lambda_{n-q}}=e^{\log (n+1) \frac{\lambda_{n-q}}{\inf _{X} \lambda_{n-q}}} \geq n+1$ by $(2.1)$.

The following special case of Proposition 2.2 is of particular interest in complex geometry.

Corollary 2.3. The following statements are equivalent:

(1) $L$ is $(n-1)$-positive;

(2) there exists a smooth Hermitian metric $h$ on $L$ and a Hermitian metric $\omega$ on $X$ such that the function

$$
\operatorname{tr}_{\omega}(-\sqrt{-1} \partial \bar{\partial} \log h)>0 .
$$

Remark 2.4. The function $\operatorname{tr}_{\omega}(-\sqrt{-1} \partial \bar{\partial} \log h)$ is globally defined on $X$ and it is independent of the choice of coordinates. It is also called the scalar curvature of the Chern curvature $R^{(L, h)}=-\sqrt{-1} \partial \bar{\partial} \log h$ with respect to the Hermitian metric $\omega$.

3. The pseudo-effective line bundles on compact complex manifolds

A line bundle $L$ on a compact complex manifold $X$ is called pseudo-effective if there exists a (possibly) singular Hermitian metric $h$ on $L$ such that its Chern curvature $R^{(L, h)}=-\sqrt{-1} \partial \bar{\partial} \log h \geq 0$ in the sense of current. In order to describe pseudoeffective line bundles in a differential geometric setting, we introduce the Bott-Chern cohomology on $X$

$$
H_{\mathrm{BC}}^{p, q}(X):=\frac{\operatorname{Ker} d \cap \Omega^{p, q}(X)}{\operatorname{Im} \partial \bar{\partial} \cap \Omega^{p, q}(X)} .
$$

Let $\operatorname{Pic}(X)$ be the set of holomorphic line bundles over $X$. As similar as the first Chern class map $c_{1}: \operatorname{Pic}(X) \rightarrow H_{\bar{\partial}}^{1,1}(X)$, there is a first Bott-Chern class map

$$
c_{1}^{\mathrm{BC}}: \operatorname{Pic}(X) \rightarrow H_{\mathrm{BC}}^{1,1}(X) .
$$

Given any holomorphic line bundle $L \rightarrow X$ and any Hermitian metric $h$ on $L$, we define $c_{1}^{\mathrm{BC}}(L)$ to be the class of its curvature form $R^{(L, h)}=-\sqrt{-1} \partial \bar{\partial} \log h$ in $H_{\mathrm{BC}}^{1,1}(X)$ 
(modulo a constant $2 \pi$ ). A Hermitian metric $\omega$ is called a Gauduchon metric if $\partial \bar{\partial} \omega^{n-1}=0$ where $\operatorname{dim}_{\mathbb{C}} X=n$. It is proved by Gauduchon ([Gau77a]) that, in the conformal class of each Hermitian metric, there exists a unique Gauduchon metric (up to constant scaling).

Proposition 3.1. The following statements are equivalent

(1) L is pseudo-effective;

(2) For any Gauduchon metric $\omega_{\mathrm{G}}$ on $X$, one has

$$
\int_{X} c_{1}^{\mathrm{BC}}(L) \wedge \omega_{\mathrm{G}}^{n-1} \geq 0
$$

Proof. The proof is essentially contained in [Yang17, Theorem 1.1] or [Yang17, Theorem 3.4] which relies on Lamari's positivity criterion ([La99]) and an observation of Michelsohn ([Mic82]). For readers' convenience, we include a proof here.

$(1) \Longrightarrow(2)$. Suppose $L$ is pseudo-effective, it is well-known that there exist a smooth Hermitian metric $h$ on $L$ and a real valued function $\varphi \in \mathscr{L}^{1}(X, \mathbb{R})$ such that

$$
R^{(L, h)}+\sqrt{-1} \partial \bar{\partial} \varphi \geq 0
$$

in the sense of current where $R^{(L, h)}=-\sqrt{-1} \partial \bar{\partial} \log h$. Then for any smooth Gauduchon metric $\omega_{\mathrm{G}}$, we have

$$
\begin{aligned}
\int_{X} c_{1}^{\mathrm{BC}}(L) \wedge \omega_{\mathrm{G}}^{n-1} & =\int_{X} R^{(L, h)} \wedge \omega_{\mathrm{G}}^{n-1} \\
& =\left(R^{(L, h)}+\sqrt{-1} \partial \bar{\partial} \varphi, \omega_{\mathrm{G}}^{n-1}\right) \geq 0
\end{aligned}
$$

since $\partial \bar{\partial} \omega_{G}^{n-1}=0$ and $R^{(L, h)}+\sqrt{-1} \partial \bar{\partial} \varphi \geq 0$ in the sense of current.

$(2) \Longrightarrow(1)$. We define several sets:

- $\mathscr{E}$ is the set of real $\partial \bar{\partial}$-closed $(n-1, n-1)$ forms on $X$;

- $\mathscr{V}$ is the set of real positive $\partial \bar{\partial}$-closed $(n-1, n-1)$ forms on $X$;

- $\mathscr{G}=\left\{\omega^{n-1} \mid \omega\right.$ is a Gauduchon metric $\}$.

In [Mic82, p.279-p.280], M.L. Michelsohn observed that $\mathscr{V}=\mathscr{G}$. Let $\mathscr{W}$ be the space of smooth Gauduchon metrics on $X$. We also define $\mathscr{F}: \mathscr{W} \rightarrow \mathbb{R}$ by

$$
\mathscr{F}(\omega)=\int_{X} c_{1}^{\mathrm{BC}}(L) \wedge \omega^{n-1} .
$$

Hence, by the assumption of (2), we have $\mathscr{F}(\omega) \geq 0$ for every $\omega \in \mathscr{W}$. Fix an arbitrary smooth Hermitian metric $h$ on $L$. Since $\mathscr{V}=\mathscr{G}$, for any $\partial \bar{\partial}$-closed positive $(n-1, n-1)$ form $\psi \in \mathscr{V}$, there exists a smooth Gauduchon metric $\omega$ such that $\omega^{n-1}=\psi$. Hence

$$
\int_{X} R^{(L, h)} \wedge \psi=\int_{X} R^{(L, h)} \wedge \omega^{n-1}=\int_{X} c_{1}^{\mathrm{BC}}(L) \wedge \omega^{n-1}=\mathscr{F}(\omega) \geq 0 .
$$


That means, as a functional on $\mathscr{V}, R^{(L, h)}$ is non-negative. Note that $\mathscr{V}$ is a hyperplane in $\mathscr{E}$. As proved in [La99, Lemma 3.3], by Hahn-Banach theorem, $c_{1}^{\mathrm{BC}}(L)$ is pseudoeffective, and there exists a locally integrable function $\varphi \in \mathscr{L}^{1}(X, \mathbb{R})$ such that

$$
R^{(L, h)}+\sqrt{-1} \partial \bar{\partial} \varphi \geq 0
$$

in the sense of current. That means, $L$ is pseudo-effective.

Of course, Proposition 3.1 has the following variant:

Proposition 3.2. The following statements are equivalent

(1) the dual line bundle $L^{-1}$ is not pseudo-effective;

(2) there exists a Gauduchon metric $\omega_{\mathrm{G}}$ such that

$$
\int_{X} c_{1}^{\mathrm{BC}}(L) \wedge \omega_{\mathrm{G}}^{n-1}>0 .
$$

4. The proofs of Theorem 1.4 and Theorem 1.5

In this section, we prove Theorem 1.4, Theorem 1.5 and Proposition 1.7.

The proof of Theorem 1.5. (1) $\Longrightarrow(2)$. By Corollary 2.3, there exist a smooth Hermitian metric $h$ on $L$ and a Hermitian metric $\omega$ on $X$ such that the function

$$
\operatorname{tr}_{\omega}(-\sqrt{-1} \partial \bar{\partial} \log h)>0 .
$$

Let $\omega_{\mathrm{G}}=e^{f} \omega$ be a Gauduchon metric in the conformal class of $\omega$ ([Gau77b]), then by (4.1), we obtain

$$
\operatorname{tr}_{\omega_{\mathrm{G}}} R^{(L, h)}=e^{-f} \cdot \operatorname{tr}_{\omega} R^{(L, h)}>0,
$$

where $R^{(L, h)}=-\sqrt{-1} \partial \bar{\partial} \log h$. In particular, we have

$$
\int_{X} \operatorname{tr}_{\omega_{G}} R^{(L, h)} \cdot \omega_{\mathrm{G}}^{n}=n \int_{X} R^{(L, h)} \wedge \omega_{G}^{n-1}=n \int_{X} c_{1}^{\mathrm{BC}}(L) \wedge \omega_{G}^{n-1}>0 .
$$

By Proposition 3.2, the dual line bundle $L^{-1}$ is not pseudo-effective.

$(2) \Longrightarrow(1)$. If $L^{-1}$ is not pseudo-effective, by Proposition 3.2, there exists a Gauduchon metric $\omega_{\mathrm{G}}$ such that

$$
\int_{X} c_{1}^{\mathrm{BC}}(L) \wedge \omega_{\mathrm{G}}^{n-1}>0 .
$$

We shall use Gauduchon's conformal trick ([Gau77a, Gau84], see also [Yang16, Yang17]) to construct a smooth Hermitian metric $h$ on $L$ such that $\operatorname{tr}_{\omega_{\mathrm{G}}}(-\sqrt{-1} \partial \bar{\partial} \log h)>0$. Hence, by Corollary 2.3, $L$ is $(n-1)$-positive.

Fix a smooth Hermitian metric $h_{0}$ on $L$. Let

$$
R_{0}=-\sqrt{-1} \partial \bar{\partial} \log h_{0}
$$


be the Chern curvature of $\left(L, h_{0}\right)$. It is easy to see that

$$
\int_{X} \operatorname{tr}_{\omega_{G}} R_{0} \cdot \omega_{\mathrm{G}}^{n}=n \int_{X} R_{0} \wedge \omega_{G}^{n-1}=n \int_{X} c_{1}^{\mathrm{BC}}(L) \wedge \omega_{G}^{n-1}
$$

Since $\omega_{\mathrm{G}}$ is Gauduchon, i.e. $\partial \bar{\partial} \omega_{\mathrm{G}}^{n-1}=0$ and the integration

$$
\int_{X}\left(\operatorname{tr}_{\omega_{G}} R_{0}-\frac{n \int_{X} c_{1}^{\mathrm{BC}}(L) \wedge \omega_{G}^{n-1}}{\int_{X} \omega_{G}^{n}}\right) \omega_{G}^{n}=0
$$

the equation

$$
\operatorname{tr}_{\omega_{G}} \sqrt{-1} \partial \bar{\partial} f=\operatorname{tr}_{\omega_{G}} R_{0}-\frac{n \int_{X} c_{1}^{\mathrm{BC}}(L) \wedge \omega_{G}^{n-1}}{\int_{X} \omega_{G}^{n}}
$$

has a solution $f \in C^{\infty}(X)$ which is well-known(e.g. [Gau77a] or [CTW16, Theorem 2.2]). Let $h=e^{f} \cdot h_{0}$ be a smooth Hermitian metric on $L$. The Hermitian line bundle $(L, h)$ has Chern curvature

$$
R^{(L, h)}=-\sqrt{-1} \partial \bar{\partial} \log h=R_{0}-\sqrt{-1} \partial \bar{\partial} f .
$$

The scalar curvature of $R^{(L, h)}$ with respect to $\omega_{\mathrm{G}}$ is

$$
\operatorname{tr}_{\omega_{\mathrm{G}}} R^{(L, h)}=\operatorname{tr}_{\omega_{G}} R_{0}-\operatorname{tr}_{\omega_{G}} \sqrt{-1} \partial \bar{\partial} f=\frac{n \int_{X} c_{1}^{\mathrm{BC}}(L) \wedge \omega_{G}^{n-1}}{\int_{X} \omega_{G}^{n}}>0 .
$$

The proof of Theorem 1.5 is completed.

By Corollary 2.3, Proposition 3.2 and Theorem 1.5, we obtain

Theorem 4.1. Let $L$ be a line bundle over a compact complex manifold $X$ with $\operatorname{dim}_{\mathbb{C}} X=n$. The following statements are equivalent:

(1) The dual line bundle $L^{-1}$ is not pseudo-effective;

(2) There exists a smooth Gauduchon metric $\omega_{\mathrm{G}}$ on $X$ such that

$$
\int_{X} c_{1}^{\mathrm{BC}}(L) \cdot \omega_{\mathrm{G}}^{n-1}>0
$$

(3) There exist a smooth Hermitian metric $h$ on $L$ and a Hermitan metric $\omega$ on $X$ such that the scalar curvature of the Chern curvature $R^{(L, h)}=-\sqrt{-1} \partial \bar{\partial} \log h$ with respect to $\omega$ is positive, i.e.

$$
s=\operatorname{tr}_{\omega} R^{(L, h)}>0
$$

(4) $L$ is $(n-1)$-positive.

It is easy to see that Theorem 4.1 has the following version on Bott-Chern classes:

Theorem 4.2. Let $X$ be a compact complex manifold $X$ with $\operatorname{dim}_{\mathbb{C}} X=n$ and $[\alpha] \in H_{\mathrm{BC}}^{1,1}(X)$. The following statements are equivalent:

(1) The class $-[\alpha]_{\mathrm{BC}}$ is not pseudo-effective; 
(2) There exists a smooth Gauduchon metric $\omega_{\mathrm{G}}$ on $X$ such that

$$
\int_{X}[\alpha]_{\mathrm{BC}} \cdot \omega_{\mathrm{G}}^{n-1}>0
$$

(3) There exist a smooth $(1,1)$ form $\chi \in[\alpha]_{\mathrm{BC}}$ and a Hermitian metric $\omega$ on $X$ such that

$$
\operatorname{tr}_{\omega} \chi>0
$$

(4) $[\alpha]_{\mathrm{BC}}$ is $(n-1)$-positive.

Now we are ready to prove our main theorem.

The proof of Theorem 1.4. The proof follows from Theorem 1.5 and a simple argument by the Serre duality.

Since $L$ is $(n-1)$-ample, by Definition 1.1 , for any ample line bundle $A$, there exists a positive number $m_{0}=m_{0}\left(K_{X} \otimes A^{-1}\right)$ such that when $m>m_{0}$, we have

$$
H^{n}\left(X, K_{X} \otimes A^{-1} \otimes L^{m}\right)=0
$$

which is also equivalent to

$$
H^{0}\left(X, A \otimes L^{-m}\right)=0
$$

by the Serre duality.

We argue by contradiction, i.e. suppose $L$ is $(n-1)$-ample, but $L$ is not $(n-1)$ positive. In this case, by Theorem 1.5 , we know the dual line bundle $L^{-1}$ must be pseudo-effective. For the pseudo-effective line bundle $L^{-1}$, it is well-known that, there exists an ample line bundle $A$ on $X$ such that for every positive integer $m$

$$
H^{0}\left(X, A \otimes L^{-m}\right) \neq 0 .
$$

This contradicts with (4.5).

For readers' convenience, we present a sketched analytical proof of (4.6) following [DPS96, Proposition 1.5]. Indeed, for a fixed very ample line bundle $H$, we choose an ample line bundle $A$ such that $A \otimes K_{X}^{-1} \otimes H^{-n}$ is also ample. Hence, there exists a positive rational number $\varepsilon_{0}$, such that for all $m \geq 0$ and rational number $\varepsilon \in\left(0, \varepsilon_{0}\right)$,

$$
c_{1}\left(L^{-m} \otimes A \otimes K_{X}^{-1} \otimes H^{-(n+\varepsilon)}\right)
$$

lies in the interior of the effective cone of $X$. It implies that $L^{-m} \otimes A \otimes K_{X}^{-1} \otimes H^{-(n+\varepsilon)}$ is linearly equivalent to an effective $\mathbb{Q}$-divisor $D$ plus a numerically trivial line bundle $T$. Hence

$$
\mathcal{O}_{X}\left(L^{-m} \otimes A\right) \simeq \mathcal{O}_{X}\left(K_{X} \otimes H^{(n+\varepsilon)} \otimes D \otimes T\right)
$$


Now, fix a point $x_{0} \notin D$. Let $\left\{s_{j}\right\} \subset H^{0}(X, H)$ be a basis such that all $s_{j}$ vanish at point $x_{0}$. Fix a local holomorphic basis $e_{H}$ of $H$ and write $s_{j}=h_{j} e_{H}$. Then

$$
h=\frac{1}{\left(\sum_{j}\left|h_{j}\right|^{2}\right)^{n}}
$$

is a singular Hermitian metric on $H^{n}$ with semi-positive curvature in the sense of current. Moreover, the weight function of $h$ is not integrable at point $x_{0}$ and the Lelong number of the curvature current is $\geq n$. On the other hand, we can put a singular metric $h_{\varepsilon}$ on $H^{\varepsilon} \otimes D \otimes T$ such that the curvature current of $h_{\varepsilon}$ equals $\varepsilon \omega+[D]$ where $\omega$ is a Kähler form in $c_{1}(H)$ and $[D]$ is the current of integration over $D$. Since $x_{0} \notin D$, the weight function of the singular metric $h h_{\varepsilon}$ on $H^{(n+\varepsilon)} \otimes D \otimes T$ has isolated singularity at point $x_{0}$. Moreover,

$$
-\sqrt{-1} \partial \bar{\partial} \log \left(h h_{\varepsilon}\right) \geq \varepsilon \omega
$$

in the sense of current and the weight function of $h h_{\varepsilon}$ has Lelong number $\geq n$ at point $x_{0}$. By Hörmander's $L^{2}$ existence theorem (e.g. [Dem90, Corollary 3.3]), we know

$$
H^{0}\left(X, K_{X} \otimes H^{(n+\varepsilon)} \otimes D \otimes T\right) \neq 0 .
$$

By (4.7), we obtain (4.6). The proof of Theorem 1.4 is completed.

The proof of Proposition 1.7. Let $f: Y \rightarrow X$ be the inclusion map. Using the projection formula and the Leray spectral sequence, one has

$$
H^{i}\left(Y, \mathcal{F} \otimes\left(f^{*} L\right)^{\otimes m}\right)=H^{i}\left(X, f_{*}(\mathcal{F}) \otimes L^{\otimes m}\right)
$$

Hence, if $L \rightarrow X$ is $q$-ample, $f^{*} L \rightarrow Y$ is also $q$-ample. On the other hand, since $\operatorname{dim}_{\mathbb{C}} Y=q+1$ and by Theorem 1.4, the $q$-ample line bundle $\left.L\right|_{Y}$ is $q$-positive.

\section{References}

[AG62] Andreotti, A.; Grauert, H. Théorème de finitude pour la cohomologie des espaces complexes. Bull. Soc. Math. France 90 (1962), 193-259.

[AV65] Andreotti, A.; Vesentini, E. Carleman estimates for the Laplace-Beltrami equation on complex manifolds. Inst. Hautes tudes Sci. Publ. Math. No. 251965 81-130.

[Bro12] Brown, M.-V. Big q-ample line bundles. Compos. Math. 148 (2012), no. 3, 790-798.

[BDPP13] Boucksom, S.; Demailly, J.-P.; Paun, M.; Peternell, P. The pseudoeffective cone of a compact Kähler manifold and varieties of negative Kodaira dimension. J. Algebraic Geom. 22 (2013) 201-248.

[CTW16] Chu, J.-C.; Tosatti, V.; Weinkove, B. he Monge-Ampère equation for non-integrable almost complex structures. arXiv:1603.00706. To appear in J. Eur. Math. Soc.

[Dem90] Demailly, J.-P. Singular hermitian metrics on positive line bundles, Proceedings of the Bayreuth conference Complex algebraic varieties, April 2-6, 1990, edited by K. Hulek, T. Peternell, M. Schneider, F. Schreyer, Lecture Notes in Math. 1507 Springer-Verlag (1992) 
[DPS96] Demailly, J.-P.; Peternell, T.; Schneider, M. Holomorphic line bundles with partially vanishing cohomology. Proceedings of the Hirzebruch 65 Conference on Algebraic Geometry (Ramat Gan, 1993), 165-198, Israel Math. Conf. Proc., 9, Bar-Ilan Univ 1996.

[Dem11] Demailly, J.-P. A converse to the Andreotti-Grauert theorem. Ann. Fac. Sci. Toulouse Math. (6) 20 (2011), Fascicule Special, 123-135.

[Gau77a] Gauduchon, P. Le théorème de l'excentricitè nulle. C. R. Acad. Sci. Paris Sér. A-B 285 (1977), no. 5, A387-A390.

[Gau77b] Gauduchon, P. Fibrés hermitiens à endomorphisme de Ricci non-négatif, Bull. Soc. Math. France 105 1977), 113-140.

[Gau84] Gauduchon, P. La 1-forme de torsion d'une varietè ehermitienne compacte. Math. Ann. 267 (1984), no. 4, 495-518.

[GK15] Greb, D.; Küronya, A. Partial positivity: geometry and cohomology of $q$-ample line bundles. Recent advances in algebraic geometry, 207-239, London Math. Soc. Lecture Note Ser., 417, Cambridge Univ. Press, Cambridge, 2015.

[Kür10] Küronya, A. Positivity on subvarieties and vanishing of higher cohomology. Ann. Inst. Fourier. Grenoble 63 (2013) 1717-1737.

[La99] Lamari, A. Courants kähleriens et surfaces compactes. Ann. Inst. Fourier 49(1999), 263285.

[Mat13] Matsumura, S. Asymptotic cohomology vanishing and a converse to the AndreottiGrauert theorem on surfaces. Ann. Inst. Fourier. Grenoble 63 (2013) 2199-2221.

[Mic82] Michelsohn, M. L. On the existence of special metrics in complex geometry. Acta Math. 149 (1982), 261-295.

[Ott12] Ottem, J.-C. Ample subvarieties and q-ample divisors. Adv. Math. 229 (2012), no. 5, 2868-2887.

[Tot13] Totaro, B. Line bundles with partially vanishing cohomology. J. Eur. Math. Soc. 15 (2013) 731-754.

[Yang16] Yang, X.-K. Hermitian manifolds with semi-positive holomorphic sectional curvature. Math. Res. Lett. 23 (2016), no.3, 939-952.

[Yang17] Yang, X.-K. Scalar curvature on compact complex manifolds. Trans. Amer. Math. Soc. 371 (2019), no. 3, 2073-2087.

[Yang18] Yang, X.-K. RC-positivity, rational connectedness and Yau's conjecture. Camb. J. Math. 6 (2018), 183-212.

[Yau78] Yau, S.-T. On the Ricci curvature of a compact Kähler manifold and the complex MongeAmpère equation. I. Comm. Pure Appl. Math. 31 (1978), no. 3, 339-411.

Morningside Center of Mathematics, Academy of Mathematics and, Systems Science, Chinese Academy of Sciences, Beijing, 100190, China

HCMS, CEMS, NCNIS, HLM, UCAS, Academy of Mathematics and, Systems Science, Chinese Academy of Sciences, Beijing 100190, China

E-mail address: xkyang@amss.ac.cn 